\title{
Stimulus exposure time and perceptual memory
}

\author{
WILLIAM VON HIPPEL and CHRIS HAWKINS \\ Ohio State University, Columbus, Ohio
}

\begin{abstract}
The recent explosion of research on implicit memory has facilitated the examination of perceptual and conceptual processes in the encoding of information. Nevertheless, stimulus exposure time--the amount of time that a stimulus is physically available to a perceiver's scrutiny-has received little attention. In the present paper, we examine the effect of stimulus exposure time on three implicit memory measures (word-fragment completion, perceptual identification, and general knowledge) and two explicit memory measures (graphemic cued recall and semantic cued recall). In Experiment 1, we demonstrated that increases in exposure time lead to increases in implicit perceptual memory, but not to implicit conceptual memory, when the encoding task focuses on perceptual features of the stimulus. We replicated this effect in Experiment 2 and demonstrated that increases in exposure time lead to increases in perceptual and conceptual memory when the measures are explicit. Thus, the current experiments demonstrate that manipulations of exposure time lead to dissociations in implicit, but not explicit, memory.
\end{abstract}

The past 10 years have seen an explosion of research on implicit memory, a notable consequence of which is the documentation of a variety of dissociations between and among implicit and explicit memory measures. These dissociations have suggested to some that implicit and explicit measures tap different memory systems (e.g., Tulving \& Schacter, 1990; Tulving, Schacter, \& Stark, 1982), and to others that the procedures are sensitive to different cognitive processes at encoding (e.g., Jacoby, 1983; Roediger, 1990). According to the processing perspective, it is not the implicit versus explicit nature of the memory measure that determines performance, but rather the match between the processes engaged at study and the processes engaged at test (cf. Blaxton, 1989; Morris, Bransford, \& Franks, 1977). Thus, memory measures have been classified as perceptually driven or conceptually driven, depending on the extent to which they are sensitive to prior perceptual or conceptual processing. For example, word-fragment completion and perceptual identification seem to be perceptually driven memory measures, because priming effects with these measures are attenuated when the modality or typeface of the words is changed between study and test (Blaxton, 1989; Jacoby \& Hayman, 1987; Roediger \& Blaxton, 1987). Conversely, category accessibility and general knowledge have been classified as conceptually driven memory measures, because priming effects

\footnotetext{
We would like to thank John Jonides and Mary Sue Weldon for useful discussions concerning these experiments, and Neal Johnson, John Jonides, Ritske De Jong, and Sunny Ginger for their helpful comments on an earlier draft of the manuscript. We would also like to thank our three reviewers, Tram Neill, Bill Johnston, and Lisa Fournier, for their constructive suggestions. Experiment 1 was presented at the 1992 meeting of the Midwestern Psychological Association, Chicago, IL. Correspondence should be addressed to W. von Hippel, Department of Psychology, Ohio State University, 1827 Neil Avenue, Columbus, OH 43210 (e-mail: vonhippel@osu.edu).
}

with these measures are facilitated by semantic processing at encoding (Blaxton, 1989; Smith \& Branscombe, 1988). Such results have been interpreted as evidence that perceptual identification and word-fragment completion are primarily measures of the amount of perceptual processing that occurs at encoding, whereas category accessibility and general knowledge are primarily measures of the amount of conceptual processing that occurs at encoding (see Roediger, 1990).

If word-fragment completion and perceptual identification are sensitive to the type of perceptual processing that occurs at encoding, then the sheer amount of perceptual processing that occurs might also determine the level of priming on these measures. One limit on the amount of perceptual processing at encoding is the length of time that a stimulus is physically available to the perceiver. As stimulus exposure time increases, the perceiver should be able to extract more perceptual information from the stimulus. This is not to say that increases in exposure time are sufficient to lead to increased perceptual processing, but rather that increases in exposure time can lead to increased perceptual processing. Task demands, perceiver motivation and attention, and a variety of other factors are critical in determining whether the exposure time that is provided will be used to facilitate perceptual processing (e.g., see Hawley \& Johnston, 1991; Neill, Beck, Bottalico, \& Molloy, 1990), but certainly exposure time is a limiting factor with regard to the amount of perceptual processing that can take place.

At present, little research has been done on this issue, and the research that does exist suggests that the amount of perceptual processing at encoding may not influence priming on perceptual measures. Richardson and Bjork (1982) and Greene (1986), for example, found null effects of rehearsal time on auditory perceptual identification and word-stem completion, respectively. Because 
they varied rehearsal time and not exposure time, however, their results are inconclusive with regard to the current question. In more directly related research, Jacoby and Dallas (1981) found an effect of exposure time on recognition memory, but not on perceptual identification, and Neill et al. (1990) found an effect of exposure time on recognition, but not on word-fragment completion. It is important to note, however, that although these researchers failed to find effects of exposure time on perceptual identification and word-fragment completion, the use of exposure time was tangential to their goals, so they did not vary exposure duration across a broad spectrum of times.

The absence of effects of exposure time on implicit perceptual memory measures is inconsistent with the notion that perceptual processing requires time. A stimulus must be present for at least a minimal time period for perception to occur. Nevertheless, it is possible that implicit memory measures are sensitive only to the amount of perceptual encoding in a roughly dichotomous fashion. It may be that above a certain minimal exposure time, additional perceptual encoding does not lead to increased priming on implicit perceptual memory measures. Another possibility is that increases in exposure time offer diminishing returns in perceptual priming. Above a certain threshold, more time will allow more perceptual processing, but the perceiver will not necessarily use the additional time for that purpose. Once enough perceptual processing has occurred, allowing identification of a stimulus, the perceiver may either engage in conceptual processing in order to extract meaning, or quit attending altogether.

If increased exposure time does in fact lead to diminishing returns in perceptual processing, previous null findings may simply mean that enough perceptual processing had occurred at the shortest exposure times, so the amount of subsequent processing that occurred at the longer times was relatively small and undetectable. This reasoning may explain the null effect of exposure time in Jacoby and Dallas (1981) and Neill et al. (1990), who used relatively frequent words and relatively long exposure times of $1 \mathrm{sec}$ or more. An examination of the effects of exposure times below and up to 1 sec may provide evidence of a more direct relationship between exposure time and perceptual memory.

Although it is difficult to assess the relevance of their research to this issue, Schacter, Cooper, Delaney, Peterson, and Tharan (1991) have demonstrated that benefits from increased exposure time may accrue with complex or unfamiliar stimuli at durations longer than $1 \mathrm{sec}$. Schacter et al. presented subjects with visual objects for either single or multiple exposures and measured implicit memory through an object-decision task. With exposure durations of $5 \mathrm{sec}$, they found that multiple exposures did not facilitate priming more than a single exposure, but with exposure durations of $1 \mathrm{sec}$, multiple exposures led to significant priming and single exposures did not. Interpretation of these results with regard to the current hypothesis is hindered by the fact that rep- etition has been shown to enhance priming on implicit perceptual measures (so long as the repetitions are spaced across the stimulus list) when increases in exposure time do not (e.g., Jacoby \& Dallas, 1981). Furthermore, other researchers investigating implicit memory for visual patterns have failed to find increases in implicit memory with increases in exposure duration beyond $1 \mathrm{sec}$ (Musen, 1991). Nevertheless, because Schacter et al. found that repetition facilitated implicit memory with 1 -sec exposures and not with 5 -sec exposures, it seems possible that these findings might implicate exposure duration as well as repetition. Thus, these findings suggest that, depending on task difficulty and the nature of the memory task, priming effects may be sensitive to changes in exposure durations over a broad range of times.

The goal of the current paper is to provide evidence that increases in stimulus exposure time lead to facilitation on implicit perceptual memory measures. In order to test this hypothesis, two experiments were conducted; subjects were exposed to words for different durations, and memory for these words was examined. In order to differentiate between the effects of exposure time on perceptual and conceptual memory, some of the subjects were given perceptual memory measures and some were given conceptual memory measures. Additionally, in Experiment 1, some of the subjects were given a perceptual encoding task and some were given a conceptual encoding task on their initial encounter with the words. It seemed likely that a perceptual orienting task would increase the subjects' sensitivity to exposure time on perceptual memory measures, because exposure time would determine the amount of perceptual processing. It was not clear, however, whether a relationship between exposure time and perceptual memory would emerge when the subjects engaged in a conceptual orienting task, because visual processing of the words might still be highly constrained by exposure time. For this reason, we predicted that increases in exposure time would facilitate perceptual memory when the encoding task required perceptual processing and, possibly, when it required conceptual processing. In contrast, we predicted that increases in exposure time would facilitate conceptual memory only when the encoding task required conceptual processing, because only under these conditions would conceptual processing be related to exposure time.

Although these predictions were expected to be upheld with the implicit memory measures of perceptual identification, word-fragment completion, and general knowledge, it was not clear whether they would describe the effects of exposure time on explicit perceptual and conceptual memory measures. Because increases in exposure time have been shown to facilitate the conscious use of memory (Debner \& Jacoby, 1994), and because explicit memory measures rely on conscious retrieval of information from memory, it seemed that explicit memory measures may be sensitive to increases in exposure time independently of whether the measures were conceptual or perceptual in nature. That is, despite the fact 
that various explicit memory measures have been demonstrated to be differentially sensitive to prior perceptual versus conceptual processing (e.g., see Blaxton, 1989), increases in exposure time were expected to facilitate both conceptual and perceptual explicit memory performance because they facilitate conscious memory processes. Consequently, the current experiments on exposure time were expected to show dissociations between conceptual and perceptual implicit measures, but not between conceptual and perceptual explicit measures. Such a finding would demonstrate that the implicit/explicit distinction is an important one even within the constraints of a "transfer-appropriate processing" framework, due to the role played by conscious retrieval strategies in explicit, but not implicit, memory.

\section{EXPERIMENT 1}

\section{Method}

Subjects. One hundred nineteen introductory psychology students, approximately half males and half females, participated in partial fulfillment of their course requirements. The subjects were between the ages of 18 and 25, were native speakers of English, and had normal or corrected-to-normal vision. Forty subjects were randomly assigned to each of the perceptual identification and general knowledge conditions, with 20 subjects engaged in the perceptual and conceptual orienting tasks within each memory measure. Thirty-nine subjects were randomly assigned to the word-fragment completion conditions; 19 of these were engaged in the perceptual orienting task and 20 were engaged in the conceptual orienting task.

Materials. Ninety words, between five and nine letters long, were selected from a list pretested and normed by Blaxton (1985). Word fragments and general-knowledge questions associated with these words were selected from the same source. See Table 1 for examples of study words, word fragments, and general-knowledge questions.

Design. The design was a 2 (study task: perceptual, conceptual) $\times 3$ (memory test: word-fragment completion, perceptual identification, general knowledge) $\times 5$ (exposure time: $50,100,200$, $500,1,000 \mathrm{msec}$ ) $\times 2$ (letter case: upper, lower). The first two factors were varied between subjects, and the latter two factors were varied within subjects.

Procedure. The subjects were run individually in cubicles, in which computers displayed the instructions and experimental materials. The computers randomly assigned each subject to one of the study conditions and one of the memory test conditions. In both of the study conditions, the subjects were told that they would be presented with words that would be exposed for varying durations. In the perceptual study conditions, they were told that for each word they would be asked to make a judgment on a 9-point scale about how easy or difficult the word was to spell, on the basis of how well the sound of the word matched its spelling. In the conceptual study conditions, the subjects were told that for each word they would be asked to make a judgment on a 9-point scale about whether the word represented a thing that belonged to the natural world or a thing that belonged to the human world. They were further told that things that were artificial should be designated as belonging to the human world, and things that were not made by humans should be designated as belonging to the natural world.

Sixty words were then randomly selected for each subject from a larger list of 90 words for presentation during this study phase of the experiment. From this study list, 12 words were randomly assigned to be shown at each of five exposure times: $50,100,200$, 500 , and $1,000 \mathrm{msec}$. Half of the words shown at each exposure time were presented in uppercase, and half were presented in lowercase. The words were presented in random order; they were preceded by 5 buffer words to reduce primacy effects and followed by 5 buffer words to reduce recency effects. These buffer words were shown for each of the five exposure times.

Two hundred fifty milliseconds before the onset of each word, 1-in. horizontal lines appeared above and below the center point of the computer screen to indicate where the word would appear. The words were then presented for the predetermined exposure time, and then they were backward masked by a string of ampersands. The backward mask was presented immediately after the stimulus words and was used to terminate the presentation of the stimulus words. A 9-point scale was presented $500 \mathrm{msec}$ after each word was masked and erased, and the subjects were required to enter an appropriate number (to indicate their response to the perceptual or conceptual study task) before the computer presented the next word. The luminance of the study words ranged from 3.8 to $4.9 \mathrm{~lx}$, depending on word length, and the luminance of the backward mask was $7.8 \mathrm{~lx}$.

Once the subjects finished viewing the 60 study words and 10 buffer words, they were presented with one of three implicit memory tests. The solutions to all three of these implicit memory tests were the 60 words that the subjects had seen during the first phase of the experiment and the 30 words, from the same stimulus list, that they had not seen earlier in the experiment. The order of the 60 target and 30 foil words was randomized for each subject.

In the word-fragment completion condition, each word fragment was presented in lowercase in the center of the screen and was surrounded by the horizontal lines that had been presented during the study phase. The cursor was located directly below the first letter of each word fragment, and the subjects were instructed to type an English word that matched the pattern of letters and blanks and then press the return key. After they entered their re-

Table 1

Examples of Study Words, Word Fragments, and General-Knowledge Questions Used in Experiments 1 and 2

\begin{tabular}{|c|c|c|}
\hline $\begin{array}{l}\text { Study } \\
\text { Words }\end{array}$ & $\begin{array}{c}\text { Word } \\
\text { Fragments }\end{array}$ & General-Knowledge Questions \\
\hline $\begin{array}{l}\text { architect } \\
\text { barrel } \\
\text { cheetah } \\
\text { eagle } \\
\text { hydrogen } \\
\text { marble } \\
\text { prison } \\
\text { purple } \\
\text { sandal } \\
\text { turkey }\end{array}$ & $\begin{array}{l}\text { a_ch__ct } \\
\text { ba___ } \\
\text { e___t_h } \\
\text { e_g_e } \\
\text { h_dr_n } \\
\text { ma_t_ } \\
\text { pr_s_- } \\
\text {-u_p_e } \\
\text { s_n_al } \\
\text { t__key }\end{array}$ & $\begin{array}{l}\text { What was Frank Lloyd Wright's profession? } \\
\text { What does a cooper make? } \\
\text { What is the fastest animal on earth? } \\
\text { What was the name of Armstrong and Aldrin's lunar module? } \\
\text { What is the most abundant element in the sun? } \\
\text { What is the Taj Mahal made of? } \\
\text { What is another name for a state penitentiary? } \\
\text { What is the most common color of amethyst? } \\
\text { What does the Statue of Liberty wear on her feet? } \\
\text { What is the heaviest land bird in North America? }\end{array}$ \\
\hline
\end{tabular}


sponse, the screen was cleared, and $500 \mathrm{msec}$ later the next word fragment was presented.

In the perceptual identification condition, each word was presented in lowercase in the center of the screen; it was surrounded by the horizontal lines that were presented during the study phase. Before encountering the target and foil words, the subjects were presented with 24 practice trials, in which words were exposed at ever-decreasing durations from 133 to $33 \mathrm{msec}$, with each word followed immediately by a mask of ampersands. Following these practice trials, the subjects were presented with the target and foil words at an exposure duration of $33 \mathrm{msec}$ each. After each word was shown and backward masked, they were prompted to type in the word they had just seen. The subjects were told to make a guess if they could not identify the word. After they entered their response and pressed the return key, the screen was cleared; $500 \mathrm{msec}$ later, the next word was presented.

In the general-knowledge condition, the subjects were told that they would be presented with trivia questions similar to those used in the game "Trivial Pursuit." They were instructed to respond to each question by typing in a single-word answer, and they were to guess if they did not know the answer. The computer then presented 90 general-knowledge questions, the answers to which were the 60 target and 30 foil words. After the subjects entered their response to each question and pushed the return key, the screen was cleared; $500 \mathrm{msec}$ later, the next question was presented.

In all of the memory conditions, the subjects were instructed to spend no more than $10 \mathrm{sec}$ on each question because the experimenter would consider the total time required for the task in assessing their performance. The subjects were told that if they could not generate a response within this time span, they were to push the return key to continue to the next item. After they responded to each item in the general-knowledge and perceptual identification conditions, the computer reported whether the answer was right or wrong. The subjects in the word-fragment completion condition did not receive such feedback, because the correctness of their answers was obvious. Once the subjects had completed their memory task, they were debriefed, thanked, and dismissed.

\section{Results}

Before examining the priming effects at different exposure times, it was necessary to determine whether the different implicit memory measures had different baseline (i.e., foil) completion rates. If the two perceptual measures had foil completion rates that were markedly different from that of the conceptual measure, differences in priming across these measures would be difficult to interpret. In order to determine whether the different implicit measures had significantly different foil completion rates, an analysis of variance (ANOVA) was conducted on the foil items across the three measures. The ANOVA revealed a significant effect for type of measure, with perceptual identification showing higher foil completion rates (.42) than either word-fragment completion $(.22)$ or general knowledge $(.18)[F(2,113)=$ $\left.22.24, M S_{\mathrm{e}}=.03, p<.001\right]$. No differences in foil completion rates emerged as a function of study task $\left[F(1,113)=1.93, M S_{\mathrm{e}}=.03, p>.15\right]$. An unexpected interaction emerged between study task and memory measure - the perceptual identification foil completion rate was higher in the conceptual study conditions than in the perceptual study conditions $[F(2,113)=3.68$, $M S_{\mathrm{e}}=.02, p<.03$; see Figure 1B]. A subsequent ANOVA, comparing word-fragment completion with general knowledge, revealed no significant differences in foil completion rates $\left[F(1,75)=1.57, M S_{\mathrm{e}}=.02, p>.20\right]$.

Because the foil rates between the perceptual measure of word-fragment completion and the conceptual measure of general knowledge were not significantly different, the priming rates from these two measures are directly comparable. Furthermore, because perceptual identification and word-fragment completion were expected not to differ with regard to their sensitivity to exposure time, it was deemed appropriate to compare their priming rates, even though their foil completion rates were vastly different. If priming rates with these two measures do differ across exposure times, then the different baseline completion rates may be a cause of this difference.

In order to determine whether increases in viewing time led to increases in perceptual memory, a series of linear and quadratic contrasts were conducted. Although our hypothesis does not specify that the increase in perceptual memory must be linear or quadratic, the presence of a linear trend would provide the most unambiguous support. We had no specific predictions concerning the quadratic contrast in the perceptual memory conditions. The results of Jacoby and Dallas (1981) suggest, however, that if there are increases in perceptual memory as a function of increases in exposure time, such increases may approach an asymptote as early as $1,000 \mathrm{msec}$. Consequently, quadratic contrasts were conducted in all conditions. A measure of priming was computed for these analyses by subtracting the percentage of correct responses made by the subjects to the foil items from the percentage of correct responses to the target items. All of the analyses were conducted with this measure of priming.

As can be seen in Figure 1, exposure time had a different impact on the perceptual and conceptual memory measures, depending on the nature of the study task. An ANOVA conducted across all three memory measures revealed a main effect for exposure time $[F(4,452)=$ $\left.17.53, M S_{\mathrm{e}}=.03, p<.001\right]$, no main effect for study task $\left[F(1,113)=.06, M S_{\mathrm{e}}=.14, p>.80\right]$, and no main effect for case $\left[F(1,113)=.00, M S_{\mathrm{e}}=.03, p>.95\right]$. Predicted interactions emerged between memory measure and exposure time $\left[F(8,452)=1.98, M S_{\mathrm{e}}=.03\right.$, $p<.05]$ and between study task and memory measure $\left[F(2,113)=3.42, M S_{\mathrm{e}}=.14, p<.04\right]$. Simple effects analyses of the latter interaction failed to reveal main effects for study task with either word-fragment completion or perceptual identification ( $p s>.15)$, although in both cases perceptual study led to slightly greater priming than conceptual study (.246 vs. .204 with wordfragment completion, and .184 vs. .135 with perceptual identification). A simple main effect for study task did emerge with general knowledge, however, in such a way that conceptual study led to greater priming $(.148)$ than perceptual study $(.073)\left[F(1,113)=3.99, M S_{\mathrm{e}}=.01\right.$, $p<.05]$.

A marginal three-way interaction also emerged between memory measure, exposure time, and letter case 

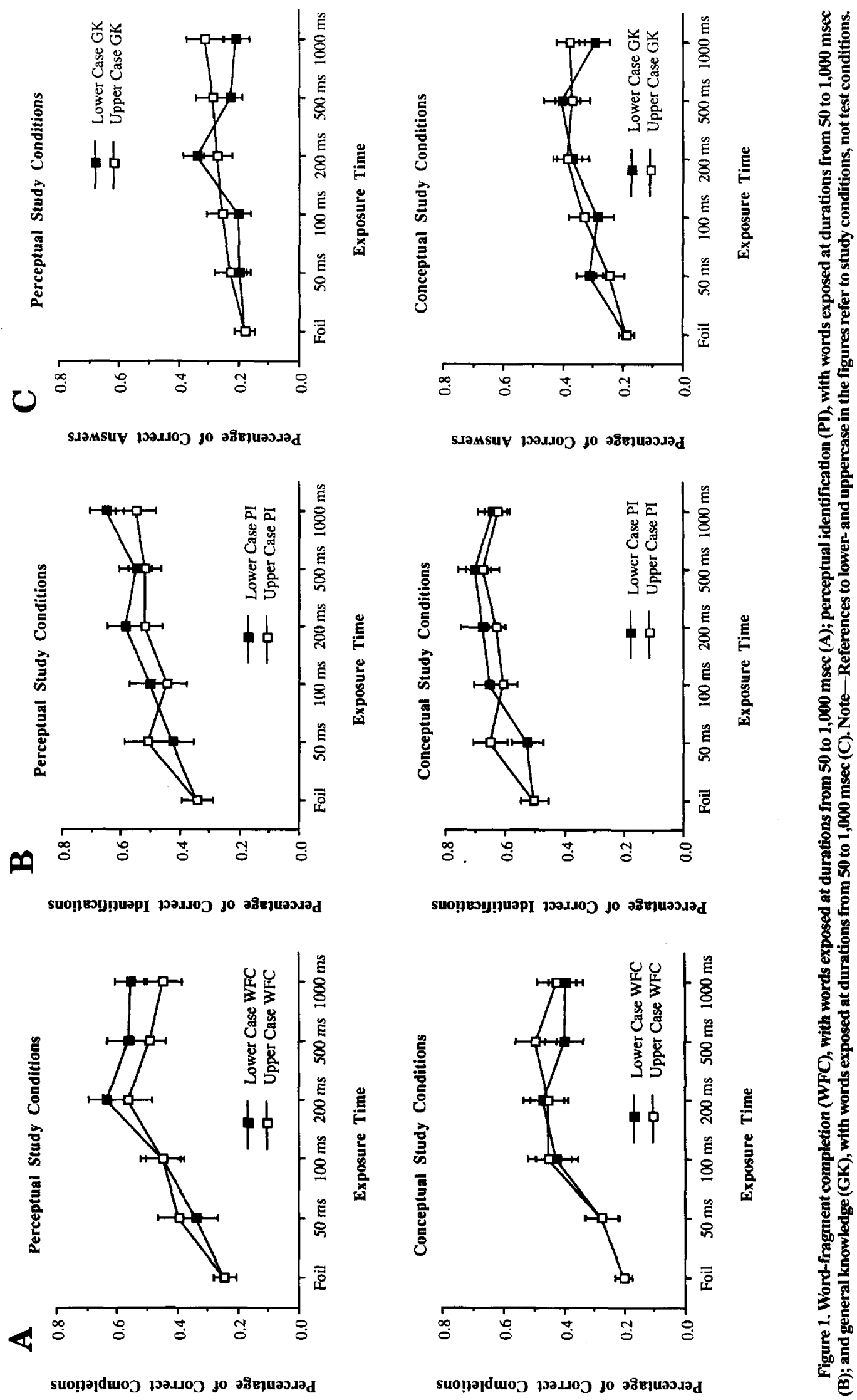
$\left[F(8,452)=1.69, M S_{\mathrm{e}}=.03, p<.10\right]$. No other interactions were significant (all $p s>10$ ). Comparisons between completion rates of targets and foils revealed significant priming in all three memory conditions $\left[F(1,38)=119.74, M S_{\mathrm{e}}=.01, p<.001\right.$ with wordfragment completion; $F(1,39)=82.58, M S_{\mathrm{e}}=.01, p<$ .001 with perceptual identification; and $F(1,39)=31.60$, $M S_{\mathrm{e}}=.01, p<.001$ with general knowledge].

A polynomial contrast conducted across all three memory measures revealed a linear trend for exposure time $[t(118)=5.94, p<.001]$ as well as a quadratic trend $[t(118)=-5.14, p<.001]$. There was no interaction between memory measure and the linear trend for exposure time $[t(118)=1.34, p>.15]$ and no interaction between study condition and the linear trend for exposure time $[t(118)=-.76, p>.40]$. There was no three-way interaction between memory measure, study condition, and the linear trend for exposure time $[t(118)=-.12, p>.90]$. Letter case did not interact with the linear trend for exposure time, either in isolation or in combination with the type of memory measure or study task (all $p s>.10$ ).

In the perceptual study conditions, a polynomial contrast revealed the predicted linear trend $[t(19)=3.08$, $p<.01]$ and a quadratic trend $[t(19)=-3.72, p<.01]$ with word-fragment completion; the predicted linear trend $[t(20)=3.64, p<.01]$ and no quadratic trend $[t(20)=0.30, p>.70]$ with perceptual identification; and no linear trend $[t(20)=1.29, p>.20]$ or quadratic trend $[t(20)=-1.28, p>.20]$ with general knowledge. ${ }^{1}$ Word-fragment completion, perceptual identification, and general knowledge did not show an effect for letter case $[t(19)=1.42, p>.10 ; t(20)=1.44, p>.10$; and $t(20)=1.23, p>.20$, respectively]. There was no interaction between letter case and the linear trend for exposure time with word-fragment completion $[t(19)=1.70$, $p>.10]$ or general knowledge $[t(20)=0.60, p>.50]$, but an interaction did emerge with perceptual identification $[t(20)=2.33, p<.05$; see Figure 1B].

In the conceptual study conditions, a polynomial contrast revealed a linear trend $[t(20)=2.35, p<.03]$ and a quadratic trend $[t(20)=-4.35, p<.001]$ with wordfragment completion; no linear trend $[t(20)=1.64, p>$ $.10]$ or quadratic trend $[t(20)=-1.56, p>.10]$ with perceptual identification; and a significant linear trend $[t(20)=2.70, p<.02]$ and a marginal quadratic trend $[t(20)=-1.83, p<.09]$ with general knowledge. Wordfragment completion, perceptual identification, and general knowledge did not show an effect for letter case $[t(20)=1.23, p>.20 ; t(20)=0.05, p>.90 ; t(20)=$ $0.36, p>.70$, respectively], and none of these measures showed an interaction between letter case and the linear trend for exposure time $[t(20)=0.91, p>.30 ; t(20)=$ $1.58, p>.10$; and $t(20)=1.41, p>.10$, respectively].

\section{Discussion}

The results of Experiment 1 provide support for the hypothesis that increases in stimulus exposure time lead to increases in perceptual memory. When the subjects engaged in perceptual study, increases in exposure time led to significant facilitation on word-fragment completion and perceptual identification, but not on general knowledge. In contrast to these results, when the subjects engaged in conceptual study, increases in exposure time led to significant facilitation on general knowledge and word-fragment completion, but not on perceptual identification. This sensitivity of word-fragment completion, but not of perceptual identification, to increases in exposure time with conceptual study may reflect different sensitivity, different baseline completion rates, or the fact that word-fragment completion seems to contain a larger conceptual component than perceptual identification (Weldon, 1991).

The differential sensitivity of the conceptual and perceptual memory measures to the effects of conceptual versus perceptual processing at encoding is consistent with the principles of transfer-appropriate processing as well as with our predictions regarding the effects of exposure time on these two types of memory measures. We proposed that a study task that led to conceptual processing at encoding would cause general knowledge to become associated with exposure time, because increases in exposure time would lead to commensurate increases in the amount of conceptual study. We proposed that a study task that led to perceptual processing at encoding, on the other hand, would cause word-fragment completion and perceptual identification to become more highly associated with exposure time, because increases in exposure time would lead to commensurate increases in the amount of perceptual study.

Somewhat surprisingly, the only effect of the letter case manipulation in Experiment 1 was the single interaction that emerged between letter case and the linear trend for exposure time when the subjects engaged in perceptual study and then completed a perceptual identification task. Although this result is consistent with predictions, there was no effect for letter case in any of the other conditions. This failure to find a larger or more generalized effect for letter case, although contrary to predictions, is consistent with research that has typically shown only a small effect for letter case on implicit perceptual memory measures (Richardson-Klavehn \& Bjork, 1988; see also Rajaram \& Roediger, 1993).

At this point, it seems worth addressing the possibility that the implicit memory measures in the current experiments may not have been treated as truly implicit by the subjects. Although $33 \%$ of the test words were not presented at study, it is possible that the subjects might have noticed that many of the test words were also study words, and consequently might have relied on intentional uses of memory to facilitate their implicit memory performance. In order to determine whether reliance on intentional retrieval strategies would lead to a different pattern of results, we included explicit as well as implicit conceptual and perceptual memory measures in Experiment 2. Specifically, we included word-fragment completion and general knowledge tests, as in Experiment 1 , but also graphemic cued-recall and semantic 
cued-recall tests. Examples of graphemic cues and their associated words are ASSUME/ASYLUM, CHASSIS/CHASTITY, and DAILY/DAISY; examples of semantic cues for these same words are INFIRMARY/ASYLUM, VIRGINITY/CHASTITY, and MARIGOLD/DAISY (Blaxton, 1985).

Similar to word-fragment completion and general knowledge, graphemic and semantic cued recall differ substantially in their sensitivity to prior perceptual versus conceptual processing. Graphemic cued recall is sensitive to the match in modality between study and test, whereas semantic cued recall is unaffected by study modality. Graphemic cued recall is also sensitive to the match in typeface between study and test, whereas semantic cued recall is insensitive to typeface, but is sensitive to imagery instructions at encoding (see Blaxton, 1989). Thus, although explicit tests of memory tend to give subjects greater freedom in their choice of retrieval strategies, providing them with graphemic versus semantic cues seems to constrain the memory measures to be primarily perceptual versus conceptual in nature.

In this regard, Blaxton (1989) has also demonstrated that the implicit versus explicit nature of the memory task may not be as important as whether the task relies on perceptual or conceptual features of the material. Despite this fact, we expected that the difference between perceptual and conceptual implicit memory measures would attenuate with explicit memory measures. This attenuation was expected, because explicit measures are dependent on conscious retrieval strategies, which have been shown to be sensitive to manipulations of exposure time (Debner \& Jacoby, 1994). Thus, independently of whether subjects are presented with perceptual or conceptual cues, increases in exposure time are likely to facilitate conscious retrieval and thereby facilitate explicit memory performance. Varying the nature of the retrieval cue should vary the predominance of the role played by perceptual or conceptual factors in retrieval, so differences between the measures may emerge. Such differences are just likely to be smaller than the differences that emerged with implicit memory measures.

\section{EXPERIMENT 2}

Because the letter case manipulation failed to reveal consistent differences in the relationship between perceptual memory and exposure time, a manipulation of letter case was not included in Experiment 2. Additionally, because the goal of this experiment was to explore the effect of perceptual study on implicit and explicit perceptual and conceptual memory, no conceptual study conditions were included. Rather, all the subjects engaged in a perceptual orienting task. In order to increase the generalizability of the results of Experiment 1, a new perceptual orienting task was chosen for Experiment 2. Additionally, in order to assess the reliability of the quadratic trend found in Experiment 1, a 2-sec exposure time was added to the study phase of the experiment.

In the test phase of the experiment, the subjects in the implicit memory conditions were given either a word- fragment completion task or a general-knowledge task; the subjects in the explicit memory conditions were given either a graphemic cued-recall task or a semantic cuedrecall task. In order to determine the extent to which the explicit recall cues were activating subjects' memory for the associated words, instead of simply priming the associated words, we instructed the subjects in the explicit memory conditions to enter the first word that came to mind whenever they could not remember a word associated with the cue word. As a consequence, the percentage of foil words that the subjects provided when presented with foil cues should serve as a baseline indicator of associative priming for the two cued-recall tasks.

\section{Method}

Subjects. One hundred twelve introductory psychology students, approximately half males and half females, participated in partial fulfillment of their course requirements. The subjects were between the ages of 18 and 25, were native speakers of English, and had normal or corrected-to-normal vision. Twenty-seven subjects were randomly assigned to the word-fragment completion condition, 28 were randomly assigned to the perceptual identification condition, 28 were randomly assigned to the graphemic cued-recall condition, and 29 were randomly assigned to the semantic cued-recall condition.

Materials. The words and word fragments were the same as those used in Experiment 1. In addition, the words that were graphemically or semantically related to the study words were selected from Blaxton (1985). These words served as cues in the cued recall tasks.

Design. The design was a 2 (memory measure: perceptual, conceptual) $\times 2$ (memory measure: implicit, explicit) $\times 6$ (exposure time: $50,100,200,500,1,000,2,000 \mathrm{msec}$ ). The first two factors were varied between subjects, and the last factor was varied within subjects.

Procedure. The subjects were individually tested using computers in cubicles. The computer randomly assigned each subject to the implicit and explicit, perceptual and conceptual memory test conditions. The computer selected and ordered words for the initial presentation through the same procedure that was used in Experiment 1 , with the exceptions that a 2-sec exposure time was added and all the words were presented in lowercase. These changes resulted in the presentation of 10 words at each of the six exposure times.

The subjects were told that they would be presented with words that would be exposed for varying durations. They were told that, for each word, they would be asked to make a judgment on a 5-point scale about how visually pleasing they thought the word was, on the basis of the word's size, shape, and its pattern of letters. A 5-point scale was presented immediately after each word was masked and erased, and the subjects were required to enter an appropriate number before the computer presented the next word.

In the memory phase of the experiment, the subjects in the implicit memory conditions were given word-fragment completion or general-knowledge tests, as in Experiment 1. The subjects in the explicit memory conditions were given a cued-recall task. Half of these subjects were presented with cues that were graphemically similar to the study words, and they were told to recall the word that they had seen earlier that was physically similar to the cue. The other half of these subjects were presented with cues that were semantically similar to the study words, and they were told to recall the word that they had seen earlier that was similar in meaning to the cue. The subjects in the cued-recall conditions were told that if they could not recall a word that was similar to the cue word, they were simply to generate the first word that came to mind that was similar to the cue word. 


\section{Results}

As in Experiment 1, foil rates were subjected to an ANOVA to determine whether the different implicit and explicit memory measures had different baseline completion rates. The ANOVA revealed a main effect for the implicit/explicit nature of the memory measure $\left[F(1,108)=86.44, M S_{\mathrm{e}}=.01, p<.001\right]$, a marginal main effect for the perceptual/conceptual nature of the memory measure $\left[F(1,108)=3.90, M S_{\mathrm{e}}=.01, p<.06\right]$, and no interaction between the two $[F(1,108)=.15$, $\left.M S_{\mathrm{e}}=.01, p>.65\right]$. Simple effects analyses revealed no significant effects for type of measure within the implicit conditions $[M=.209$ with word-fragment completion, $M=.170$ with general knowledge; $F(1,108)=2.36$, $\left.M S_{\mathrm{e}}=.01, p>.10\right]$ or the explicit conditions $[M=.052$ with graphemic cued recall, $M=.027$ with semantic cued recall; $\left.F(1,108)=1.55, M S_{\mathrm{e}}=.01, p>.20\right]$.

As can be seen in Figure 2, exposure time had a different impact on the perceptual and conceptual memory measures as a function of whether they were implicit or explicit. An ANOVA conducted across memory measures revealed a main effect for exposure time $[F(5,540)=$ $\left.12.70, M S_{\mathrm{e}}=.02, p<.001\right]$, no interaction between the implicit/explicit nature of the memory measure and exposure time $\left[F(5,540)=.11, M S_{\mathrm{e}}=.02, p>.95\right]$, a marginal interaction between the perceptual/conceptual nature of the memory measure and exposure time $\left[F(5,540)=1.94, M S_{\mathrm{e}}=.02, p<.09\right]$, and a marginal three-way interaction between these variables $[F(5,540)=$ $\left.2.21, M S_{\mathrm{e}}=.02, p<.06\right]$. Additionally, comparisons between completion rates of targets and foils revealed significant priming in all four memory conditions $[F(1,26)=$ $48.81, M S_{\mathrm{e}}=.01, p<.001$ with word-fragment completion; $F(1,27)=32.18, M S_{\mathrm{e}}=.01, p<.001$ with general knowledge; $F(1,27)=62.46, M S_{\mathrm{e}}=.01, p<.001$ with graphemic cued recall; and $F(1,28)=61.86$, $M S_{\mathrm{e}}=.01, p<.001$ with semantic cued recall].

A polynomial contrast conducted across memory measures revealed the predicted linear trend for exposure time $[t(111)=7.54, p<.001]$ as well as a quadratic trend $[t(111)=-2.51, p<.02]$. This analysis revealed no interaction between the implicit/explicit nature of the memory measure and the linear trend for exposure time $[t(111)=.08, p>.90]$, but it did reveal the predicted interaction between the perceptual/conceptual nature of the memory measure and the linear trend for exposure time $[t(111)=2.43, p<.02]$. A three-way interaction also emerged between the type of memory measure (implicit/explicit and perceptual/conceptual) and the linear trend for exposure time $[t(111)=2.68, p<.01]$.

In the implicit memory conditions, a linear trend emerged for exposure time $[t(54)=4.69, p<.001]$, and there was the predicted interaction between the perceptual/conceptual nature of the memory measure and the linear trend for exposure time $[t(54)=3.15, p<.01]$. There was also a marginal quadratic trend for exposure time $[t(54)=-1.77, p<.09]$. In the explicit memory conditions, there was a linear trend for exposure time $[t(56)=6.30, p<.001]$, and no interaction between the
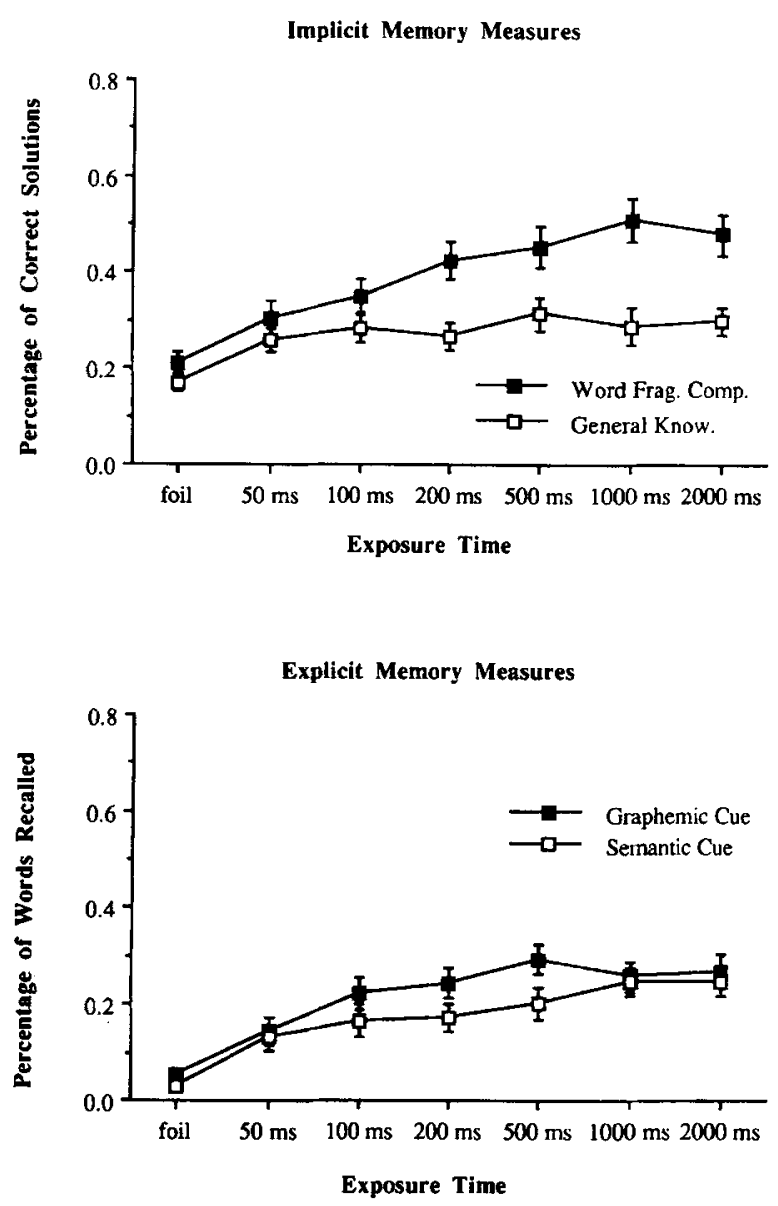

Figure 2. Word-fragment completion, general knowledge, graphemic cued recall, and semantic cued recall, with words exposed at durations from 50 to 2,000 msec.

perceptual/conceptual nature of the memory measure and the linear trend for exposure time $[t(56)=.20, p>$ $.80]$. There was also a marginal quadratic trend for exposure time $[t(56)=-1.78, p<.09]$.

To turn to an analysis by memory measure - with word-fragment completion, a polynomial contrast revealed the predicted linear trend for exposure time $[t(26)=4.91, p<.001]$ as well as a marginal quadratic trend $[t(26)=-1.97, p<.06]$; with general knowledge, a polynomial contrast revealed no linear trend for exposure time $[t(27)=1.27, p>.20]$ and no quadratic trend $[t(27)=-.53, p>.60]$; with graphemic cued recall, a polynomial contrast revealed the predicted linear trend for exposure time $[t(27)=4.50, p<.001]$ and a quadratic trend $[t(27)=-2.20, p<.04]$; and with semantic cued recall, a polynomial contrast revealed a linear trend for exposure time $[t(28)=4.43, p<.001]$ and no quadratic trend $[t(28)=-.10, \dot{p}>.90]$.

\section{Discussion}

The results of this experiment reveal important differences between perceptual and conceptual memory measures as a function of whether they are implicit or 
explicit. When the memory measures were implicit, perceptual memory increased with increases in exposure time, but conceptual memory did not. When the memory measures were explicit, perceptual and conceptual memory both increased with increases in exposure time. These results suggest that perceptual and conceptual memory measures operate somewhat differently as a function of whether they are implicit or explicit in nature. Specifically, the increases in both semantic and graphemic cued recall, which emerged as a function of increases in exposure time, suggest that increasing exposure time generally makes information more available to conscious retrieval (see also Debner \& Jacoby, 1994; Jacoby \& Dallas, 1981; Neill et al., 1990). These findings also suggest that the subjects were probably not engaging in explicit retrieval strategies in the implicit memory tasks in Experiments 1 and 2. Had they relied on explicit retrieval strategies, the cued-recall results of Experiment 2 suggest that they would have shown increases on both memory measures as a function of exposure time.

In this regard, it is worth noting that the procedure of the explicit memory tasks in Experiment 2 did not mirror the procedure of the implicit tasks. Procedural isomorphism would have been accomplished if we had presented word fragments or general-knowledge questions in the explicit conditions and asked the subjects to provide solutions from words that they recollected from earlier in the experiment. Instead of using this procedure, however, we chose to rely on the explicit tests of graphemic and semantic cued recall. This choice was motivated by two concerns. First, and most importantly, graphemic and semantic cued recall have been demonstrated to be primarily sensitive to perceptual and conceptual processing, respectively (e.g., Blaxton, 1989), whereas explicit use of word fragments and generalknowledge questions has not been studied with regard to this issue. For this reason, reliance on the cued-recall measures seemed like the more conservative choice.

Second, although researchers tend to be more concerned that implicit memory measures might lead to explicit retrieval strategies (e.g., Bowers \& Schacter, 1990), it seemed that the opposite problem might arise when using word fragments and general-knowledge questions to measure explicit memory. That is, it seemed likely that when the subjects were cued with word fragments or general-knowledge questions, their explicit memory might contain a larger implicit component than when they were cued with graphemically or semantically similar words. This concern stemmed from the expectation that the foil completion rate (i.e., the probability of generating foil words when presented with foil cues) would be substantially higher with word fragments and general-knowledge questions than with graphemic and semantic cues (see Blaxton, 1989). Because procedures that have high foil completion rates allow subjects to readily arrive at solutions without relying on a (presumably) effortful search of memory, such procedures seemed likely to encourage strategies other than reliance on explicit memory. For this reason, the graphemic and semantic cues were expected to be more appropriate than word fragments or general-knowledge questions for cuing explicit memory.

As can be seen in Figure 2, the data supported the assumption that foil completion rates would be lower for the cued-recall measures than for the implicit memory measures (indeed, the foil completion rate in the cuedrecall conditions was roughly one fifth that of the implicit memory conditions). This difference emerged even though subjects in the cued-recall conditions were instructed to generate an appropriate associate whenever they could not remember a word that was similar to the cue word. Because measures of memory are never process pure (cf. Jacoby, 1991), the best strategy in the current circumstances seemed to be to choose an explicit memory measure that would be as untainted as possible by implicit memory. Although we know of no data on this point, the difference in foil completion rates suggests that the cued-recall measures were less likely to be tainted by implicit memory than the fragment completion and general-knowledge procedures; thus, they provide a better indicator of the effect of exposure time on explicit perceptual and conceptual memory.

It is also important to note that the results of Experiment 2 provide evidence that manipulations of exposure time can lead to dissociations between implicit memory measures when such dissociations do not emerge between comparable explicit memory measures. As Blaxton (1989) has demonstrated, graphemic and semantic cued recall are sensitive to prior perceptual and conceptual processing in much the same way that word-fragment completion and general knowledge are. Nevertheless, the results from these perceptual and conceptual explicit tests did not mirror those from the perceptual and conceptual implicit tests, because only the implicit tests were differentially sensitive to the effects of exposure time. This interaction between the implicit/explicit and the perceptual/conceptual nature of the memory measures seems to implicate the role that conscious retrieval plays in explicit, but not implicit, memory. Thus, the results of Experiment 2 suggest that although explicit memory measures may mirror implicit measures in important ways (see Blaxton, 1989), the explicit/implicit distinction will remain important to any task that influences conscious components of memory.

\section{GENERAL DISCUSSION}

Overall, the current experiments demonstrate the sensitivity of perceptual memory measures to variation in stimulus exposure time. The results support the hypothesis that increases in exposure time lead to increases in perceptual memory. In Experiment 1, we explored the effect of exposure time on perceptual memory by demonstrating the impact of encoding tasks that elicited either perceptual or conceptual processing. The pattern of results with the perceptual orienting task in Experiment 1 was as predicted, with word-fragment comple- 
tion and perceptual identification showing positive linear trends with increasing exposure time. With the conceptual orienting task, on the other hand, general knowledge and word-fragment completion showed linear increases in conjunction with increases in exposure time. Additionally, Experiment 2 revealed linear increases in word-fragment completion, but not general knowledge, as a function of increasing exposure time. Experiment 2 also demonstrated linear increases in graphemic and semantic cued recall as a function of increasing exposure time.

Although the predictions from these experiments were only that increases in exposure time would lead to increases in perceptual memory, it is informative to note that quadratic trends emerged in the word-fragment completion results of Experiments 1 and 2. These results suggest that increases in exposure time may lead to decreasing marginal returns in perceptual memory, because the effects of increasing exposure time may level off at or before the duration of $1 \mathrm{sec}$. Thus, the significant quadratic trends in Experiments 1 and 2 suggest that the facilitating effects of exposure time on perceptual memory for relatively short words may primarily occur at brief durations of less than $1 \mathrm{sec}$. It seems quite possible, however, that longer or less common words might show increasing benefit from exposure times longer than 1 sec (e.g., see Schacter et al., 1991), and that highly motivated perceivers might receive increasing benefit from exposure times longer than $1 \mathrm{sec}$.

By demonstrating the sensitivity of implicit perceptual memory measures to differences in exposure time, the results of the current experiments call into question the interpretation of previous research on the effects of linguistic context on perceptual memory. For example, Levy and Kirsner (1989) and Oliphant (1983) have demonstrated that words that are read in sentences show no priming on later word-level perceptual memory measures. Similarly, MacLeod (1989) has demonstrated decreased perceptual memory for words read in a meaningful context than for words read in lists or in a nonsensical context. These experiments have been interpreted as evidence that words read in meaningful sentences and words read out of context or in lists are encoded at different linguistic levels, and that the memory task must be at the appropriate linguistic level in order to capture any effects of prior processing. In this regard, Levy and Kirsner (1989) found evidence of sentence-level implicit memory in the absence of word-level implicit memory.

In Levy and Kirsner's (1989) and Oliphant's (1983) experiments, however, words that were read in sentences surely received far less visual processing time than words read in lists. And in MacLeod's (1989) experiments, subjects were instructed to interrupt their reading to cross out words that did not fit in the context. By causing subjects to disrupt their reading and attend to words presented out of context, this task must have induced a great deal of additional visual processing time. ${ }^{2}$ Thus, exposure time has been a confounding variable in studies on the effects of linguistic levels on perceptual priming, because words presented in larger linguistic structures, such as sentences or paragraphs, are read more quickly than words presented in lists. The failure of these studies to show significant word-level priming when words were presented in sentences or paragraphs may be the result of the short exposure times at which these words were presented, and not (or to a lesser degree) the effect of the linguistic context itself on perceptual priming. For these reasons, we would be inclined to agree with Jacoby's (1983) proposal that "the effect of context is to determine the amount of visual evidence that a reader requires to be confident in his or her reading of the word" (p. 506).

Finally, the results of the current experiments provide a mechanism for the recent finding that although people have better conceptual memory for words that are encoded with a schema, they have better perceptual memory for words that are encoded without a schema (von Hippel, Jonides, Hilton, \& Narayan, 1993). It was found by von Hippel et al. that inhibitory effects of schematic processing on perceptual memory emerged whether schemata were necessary for comprehension (Bransford \& Johnson, 1973), were simply an expectation about an individual (Srull, 1981), or were the result of chronic individual differences in trait accessibility (Markus, 1977). The current results concerning exposure time suggest that because schemata (in all of their manifestations) allow a more rapid interpretation of information, they have the potential to inhibit perceptual memory even as they facilitate conceptual memory. Thus, to the extent that perceptual memory is sensitive to differences in exposure time, any cognitive process that leads to more rapid encoding of information has the potential to decrease perceptual memory for that information.

\section{REFERENCES}

Blaxton, T. A. (1985). Investigating dissociations among memory measures: Support for a transfer appropriate processing framework. Unpublished doctoral dissertation, Purdue University.

BlaxTON, T. A. (1989). Investigating dissociations among memory measures: Support for a transfer appropriate processing framework. Journal of Experimental Psychology: Learning, Memory, \& Cognition, 15, 657-668.

Bowers, J. S., \& Schacter, D. L. (1990). Implicit memory and test awareness. Journal of Experimental Psychology: Learning, Memory, \& Cognition, 16, 404-416.

Bransford, J. D., \& JohNSON, M. K. (1973). Considerations of some problems of comprehension. In W. G. Chase (Ed.), Visual information processing (pp. 383-438). New York: Academic Press.

DeBneR, J. A., \& JACOBY, L. L. (1994). Unconscious perception: Attention, awareness, and control. Journal of Experimental Psychology: Learning, Memory, \& Cognition, 20, 304-317.

Grenne, R. L. (1986). Word stems as cues in recall and completion tasks. Quarterly Journal of Experimental Psychology, 38, 663-673.

Hawley, K. J., \& Johnston, W. A. (1991). Long-term perceptual memory for briefly exposed words as a function of awareness and attention. Journal of Experimental Psychology: Human Perception \& Performance, 17, 807-815.

JACOBY, L. L. (1983). Remembering the data: Analyzing interactive processes in reading. Journal of Verbal Learning \& Verbal Behavior, 22, 485-508. 
JACOBY, L. L. (1991). A process dissociation framework: Separating automatic from intentional uses of memory. Journal of Memory \& Language, 30, 513-541.

JACOBY, L. L., \& DALLAS, M. (1981). On the relationship between autobiographical memory and perceptual learning. Journal of Experimental Psychology: General, 110, 306-340.

JACOBY, L. L., \& HaYMAN, C. A. G. (1987). Specific visual transfer in word identification. Journal of Experimental Psychology: Learning, Memory, \& Cognition, 13, 456-463.

LeVy, B. A., \& KIRSNER, K. (1989). Reprocessing text: Indirect measures of word and message level processes. Journal of Experimental Psychology: Learning, Memory, \& Cognition, 15, 407-417.

MACLEOD, C. M. (1989). Word context during initial exposure influences degree of priming in word fragment completion. Journal of Experimental Psychology: Learning, Memory, \& Cognition, 15, 398-406.

MARKUS, H. (1977). Self-schemas and processing information about the self. Journal of Personality \& Social Psychology, 35, 63-78.

MoRris, C. D., BRANSFORD, J. D., \& Franks, J. J. (1977). Levels of processing versus transfer appropriate processing. Journal of Verbal Learning \& Verbal Behavior, 16, 519-533.

MUSEN, G. (1991). Effects of verbal labeling and exposure duration on implicit memory for visual patterns. Journal of Experimental Psychology: Learning, Memory, \& Cognition, 17, 954-962.

Neill, W. T., Beck, J. L., BotTalico, K. S., \& Molloy, R. D. (1990). Effects of intentional versus incidental learning on explicit and implicit tests of memory. Journal of Experimental Psychology: Learning, Memory, \& Cognition, 16, 457-463.

Oliphant, G. W. (1983). Repetition and recency effects in word recognition. Australian Journal of Psychology, 35, 393-403.

RaJARAM, S., \& RoEdiger, H. L., III (1993). Direct comparison of four implicit memory tests. Journal of Experimental Psychology: Learning, Memory, \& Cognition, 19, 765-776.

RICHARDSON, A., \& BJORK, R. A. (1982, November). Recognition versus perceptual identification: Effects of rehearsal type and duration. Paper presented at the meeting of the Psychonomic Society, Minneapolis, $\mathrm{MN}$

RichaRdSON-KLAVEHN, A., \& BJoRK, R. A. (1988). Measures of memory. Annual Review of Psychology, 39, 475-543.

ROEDIGER, H. L., III (1990). Implicit memory: Retention without remembering. American Psychologist, 45, 1043-1056.

RoEdiger, H. L., III, \& Blaxton, T. A. (1987). Effects of varying modality, surface features, and retention interval on priming in wordfragment completion. Memory \& Cognition, 15, 379-388.
Schacter, D. L., Cooper, L. A., Delaney, S. M., Peterson, M. A., \& THARAN, M. (1991). Implicit memory for possible and impossible objects: Constraints on the construction of structural descriptions. Journal of Experimental Psychology: Learning, Memory, \& Cognition, 17, 3-19.

SMith, E. R., \& Branscombe, N. R. (1988). Category accessibility as implicit memory. Journal of Experimental Social Psychology, 24, 490-504.

SRULL, T. K. (1981). Person memory: Some tests of associative storage and retrieval models. Journal of Experimental Psychology: Human Learning \& Memory, 7, 440-463.

Tulving, E., \& Schacter, D. L. (1990). Priming and human memory systems. Science, 247, 301-305.

Tulving, E., Schacter, D. L., \& Stark, H. A. (1982). Priming effects in word-fragment completion are independent of recognition memory. Journal of Experimental Psychology: Learning, Memory, \& Cognition, 8, 336-342.

von HipPel, W., Jonides, J., Hilton, J. L., \& Narayan, S. (1993). Inhibitory effect of schematic processing on perceptual encoding. Journal of Personality \& Social Psychology, 64, 921-935.

WELDON, M. S. (1991). Mechanisms underlying priming on perceptual tests. Journal of Experimental Psychology: Learning, Memory, \& Cognition, 17, 526-541.

\section{NOTES}

1. Although the linear trend with general knowledge was not significant, inspection of the means revealed an effect that appeared to be rather orderly in the uppercase condition (see Figure 1C). For this reason, a replication of the general-knowledge condition was conducted with 40 subjects, and letter case was manipulated between subjects. This replication failed to reveal any effect of exposure time or letter case on the conceptual memory measure of general knowledge, nor did the means appear orderly, as they did in this condition.

2. By directing subjects' attention to out-of-context words, this task also had the potential to increase perceptual memory in the absence of differences in processing time that might have emerged. As Hawley and Johnston (1991) have since shown, directing attention to words facilitates implicit perceptual memory for those words, even when exposure duration is held constant.

(Manuscript received March 19, 1993; revision accepted for publication May 1, 1994.) 